\title{
Absolute continuity in periodic thin tubes and strongly coupled leaky wires
}

\author{
Francois Bentosela ${ }^{1,2}$, Pierre Duclos ${ }^{1,3}$, and Pavel Exner ${ }^{4,5}$ \\ 1 Centre de Physique Théorique, C.N.R.S., Luminy Case 907, \\ F-13288 Marseille Cedex 9, \\ 2 Université de la Mediterranée (Aix-Marseille II), F-13288 \\ Marseille, \\ 3 PhyMat, Université de Toulon et du Var, BP 132, F-83957 \\ La Garde Cedex, France; \\ 4 Department of Theoretical Physics, Nuclear Physics Institute, \\ Academy of Sciences, CZ-25068 Řež near Prague, \\ 5 Doppler Institute, Czech Technical University, Břehová 7, \\ CZ-11519 Prague, Czechia; \\ bento@cpt.univ-mrs.fr, duclos@univ-tln.fr, \\ exner@ujf.cas.cz
}

\begin{abstract}
Using a perturbative argument, we show that in any finite region containing the lowest transverse eigenmode, the spectrum of a periodically curved smooth Dirichlet tube in two or three dimensions is absolutely continuous provided the tube is sufficiently thin. In a similar way we demonstrate absolute continuity at the bottom of the spectrum for generalized Schrödinger operators with a sufficiently strongly attractive $\delta$ interaction supported by a periodic curve in $\mathbb{R}^{d}, d=2,3$.
\end{abstract}

\section{Introduction}

In models of periodically structured quantum systems, absolute continuity of the spectrum is a crucial property. For usual Schrödinger operators and many other PDE's with periodic coefficients the problem is well understood 
- see, e.g. [Ku, $\mathrm{RS}$. On the other hand, there are important classes of operators which still pose open questions. An example is represented by so-called quantum waveguides, i.e. systems the Hamiltonian of which is (a multiple of) the Laplacian in an infinitely long tube-shaped region, usually with Dirichlet boundary conditions.

In the two-dimensional setting, where the region in question is a periodically curved planar strip the absolute continuity has been demonstrated recently in [SV]. Unfortunately, the method used in this work does not seem to generalize to other dimensions including the physically interesting case of a periodic tube in $\mathbb{R}^{d}, d=3$. This is why we present in this letter a simpler result stating the absolute continuity at the bottom of the spectrum for tubes which are thin enough. With physical applications in mind we formulate it for $d=2,3$, but the argument can be used in any dimension.

We also address an analogous problem concerning Schrödinger operators in $L^{2}\left(\mathbb{R}^{d}\right), d=2,3$, with an attractive $\delta$ interaction supported by a periodic curve; we prove absolute continuity, again at the bottom of the spectrum, for a sufficiently strong attraction. If $d=2$ the answer was known for a family of curves periodic in two independent directions $\mathrm{BSS}$, however, for a single infinite curve results have been missing, to say nothing about the more singular case of dimension $d=3$.

Our method is perturbative. We show that for a sufficiently thin tube or strongly attractive $\delta$ interaction the Floquet eigenvalues do not differ much from the Floquet eigenvalues of a suitable comparison problem in one dimension which are known to be nonconstant as functions of quasimomentum. A similar argument was used recently for periodically perturbed magnetic channels [EJK], and in a different context to demonstrate existence of persistent currents in leaky quantum wire loops [EY2].

\section{Thin curved tubes}

Let $\Gamma: \mathbb{R} \rightarrow \mathbb{R}^{d}, d=2,3$, be a $C^{4}$ smooth curve without self-intersections which is periodic, i.e. there are $L>0$ and a nonzero vector $b \in \mathbb{R}^{d}$ such that

$$
\Gamma(s+L)=b+\Gamma(s), \quad \forall s \in \mathbb{R} .
$$

With an abuse of notation we will use the same symbol $\Gamma$ for the map and for the image $\Gamma(\mathbb{R})$. Let further $\Omega:=\left\{x \in \mathbb{R}^{d}\right.$ : dist $\left.(x, \Gamma)<a\right\}$ for a fixed $a>0$. If the latter is small enough, such a tube has no self-intersections and 
we can parametrize it by natural curvilinear coordinates. More specifically, take a ball $\mathcal{B}_{a}:=\left\{u \in \mathbb{R}^{d-1},|u|<a\right\}$ and consider the maps $\phi: \mathbb{R} \times \mathcal{B}_{a} \rightarrow \mathbb{R}^{d}$ defined by

$$
\begin{aligned}
\phi_{\Gamma}(s, u) & =\Gamma(s)-u n(s) \\
\phi_{\Gamma}(s, r, \vartheta) & =\Gamma(s)-r[n(s) \cos (\vartheta-\beta(s))+b(s) \sin (\vartheta-\beta(s))]
\end{aligned}
$$

for $d=2,3$, respectively, where $n(s), b(s)$ are the normal and binormal vector to $\Gamma$ at the point $s$ and the function $\beta$ in the three-dimensional case (rotation with respect to the Frenet frame) will be specified later. We will always assume that $a$ is so small that the above maps are diffeomorphisms.

The object of our interest is the Dirichlet Laplacian $-\Delta_{D}^{\Omega}$ defined conventionally [RS, Sec. XIII.15] as the self-adjoint operator associated with the quadratic form $\psi \mapsto\|\nabla \psi\|_{L^{2}(\Omega)}^{2}$ with the domain $W_{0}^{2,1}(\Omega)$.

The curvilinear coordinates allow us to "straighten" the tube, i.e. to pass to an operator on $L^{2}\left(\mathcal{C}_{a}\right)$, where $\mathcal{C}_{a}:=\mathbb{R} \times \mathcal{B}_{a}$ is a straight cylinder. The appropriate unitary operator $U: L^{2}(\Omega) \rightarrow L^{2}\left(\mathcal{C}_{a}\right)$ is at that defined by $U \psi:=$ $g^{1 / 4} \psi \circ \phi_{\Gamma}$, where $g^{1 / 2}$ is the corresponding Jacobian, $g^{1 / 2}=1+u \gamma(s)$ for $d=2$ and $g^{1 / 2}=1+r \gamma(s) \cos (\vartheta-\beta(s))$ for $d=3$, with $\gamma$ being the curvature of $\Gamma$. The price we pay for the simplification of the region is the more complicated form of the operator $H_{a, \Gamma}:=U\left(-\Delta_{D}^{\Omega}\right) U^{-1}$; a straightforward calculation ES, DE gives

$$
H_{a, \Gamma}=-\partial_{s} h^{-2} \partial_{s}-\Delta_{D}^{\mathcal{B}_{a}}+V,
$$

where $h:=g^{1 / 2}$ and $V$ is the effective potential induced by the geometry,

$$
V=-\frac{\gamma^{2}}{4 h^{2}}+\frac{h_{s s}}{2 h^{3}}-\frac{5 h_{s}^{2}}{4 h^{4}}
$$

For $d=3$ the transformation requires to choose $\beta(s)=\int_{s_{0}}^{s} \tau(s) d s$, where $\tau$ is the torsion of $\Gamma$; the latter appear also in the effective potential coming from the derivatives of $h$ by Frenet-Serret formulae [DE].

As the first step we denote $\mathcal{C}_{a}^{L}:=[0, L) \times \mathcal{B}_{a}$ and perform the usual Floquet decomposition over the Brillouin zone $\mathcal{B}:=[-\pi / L, \pi / L)$.

Lemma 2.1 There is a unitary $\mathcal{U}: L^{2}\left(\mathcal{C}_{a}\right) \rightarrow \int_{\mathcal{B}}^{\oplus} L^{2}\left(\mathcal{C}_{a}^{L}\right) d \theta$ such that

$$
\mathcal{U} H_{a, \Gamma} \mathcal{U}^{-1}=\int_{\mathcal{B}}^{\oplus} H_{a, \Gamma}(\theta) d \theta,
$$

where the fibre operator satisfies periodic b.c. in s acting as

$$
H_{a, \Gamma}(\theta)=\left(-i \partial_{s}+\theta\right) h^{-2}\left(-i \partial_{s}+\theta\right)-\Delta_{D}^{\mathcal{B}_{a}}+V .
$$


Proof: This is a classical result. We use here the modification, sometimes ascribed to Skriganov, of [RS, Thm XIII.88] with the Floquet-Bloch transform given for all $(\theta, s, u) \in \mathcal{B} \times[0, L) \times \mathcal{B}_{a}$ by the formula

$$
(\mathcal{U} f)(\theta, s, u):=\sum_{n \in \mathbb{Z}} \sqrt{\frac{L}{2 \pi}} \mathrm{e}^{-i n \theta L-i \theta s} f(s+L n, u) .
$$

An exhaustive discussion for $d=2$ can be found in $[\mathrm{YO}$, the argument for $d=3$ is analogous.

We need also the character of $\theta$-dependence of the fibre operators.

Lemma 2.2 $\left\{H_{a, \Gamma}(\theta): \theta \in \mathcal{B}\right\}$ is a type $A$ analytic family.

Proof: Splitting $H_{a, \Gamma}(\theta)$ in two pieces $H_{a, \Gamma}(0)=-\partial_{s} h^{-2} \partial_{s}-\Delta_{D}^{\mathcal{B}_{a}}+V$ and $H_{a, \Gamma}(\theta)-H_{a, \Gamma}(0)=-i \theta\left(\partial_{s} h^{-2}+h^{-2} \partial_{s}\right)+\theta^{2} h^{-2}$, one sees that the first piece is self-adjoint on $\left\{f \in W^{2,2}\left(\mathcal{C}_{a}^{L}\right), f(L, u)=f(0, u), \partial_{s} f(L, u)=\partial_{s} f(0, u)\right\}$ and that the second one is entire analytic and relatively bounded perturbation of $H_{a, \Gamma}(0)$ with zero relative bound.

Our main tool is the perturbation theory w.r.t. $a$. Since the argument follows closely $[\mathrm{DE}]$ we will just sketch it. First we use transverse scaling to pass to a unitarily equivalent operator on $L^{2}\left(\mathcal{C}_{a}^{L}\right)$ given by

$$
\tilde{H}_{a}(\theta):=\left(-i \partial_{s}+\theta\right) h_{a}^{-2}\left(-i \partial_{s}+\theta\right)-a^{-2} \Delta_{D}^{\mathcal{B}_{1}}+V_{a},
$$

where $h_{a}(s, u):=h(s, a u)$ and $h_{a}(s, r, \vartheta):=h(s, a r, \vartheta)$ for $d=2,3$, respectively, and similarly for $V_{a}$. Let $\chi_{j}$ and $\kappa_{j}^{2}$ be the eigenfuctions and eigenvalues of $-\Delta_{D}^{\mathcal{B}_{1}}$. Using this transverse basis we pass to the matrix representation,

$$
\tilde{H}_{a, j k}(\theta)=a^{-2} \kappa_{j}^{2} \delta_{j k}+T_{j k}, \quad T_{j k}:=\left(-i \partial_{s}+\theta\right)\left(h_{a}^{-2}\right)_{j k}\left(-i \partial_{s}+\theta\right)+V_{a, j k},
$$

where $f_{j k}:=\int_{-1}^{1} f(\cdot, a u) \chi_{j}(u) \chi_{k}(u) d u$ if $d=2$ and similarly for $d=3$. We need a reference operator which will be chosen in the form

$$
\tilde{H}_{a}^{0}(\theta):=I \otimes\left(-a^{-2} \Delta_{D}^{\mathcal{B}_{1}}\right)+S(\theta) \otimes I, \quad S(\theta):=\left(-i \partial_{s}+\theta\right)^{2}-\frac{1}{4} \gamma^{2}
$$

with periodic b.c. in the variable $s$. The spectrum of $S(\theta)$ is purely discrete; we denote its eigenvalues arranged in the ascending order as $\lambda_{n}(\theta)$ with the 
index $n \in \mathbb{N}$. Recall that the spectrum of $S(\theta)$ is simple with a possible exception of the endpoints of the Brillouin zone and $\theta=0$. Let $K$ be a compact subset of $\mathcal{B}$ which does not contain the points 0 and $\pm \pi / L$. The eigenvalues $\epsilon_{j n}^{0}(a, \theta):=a^{-2} \kappa_{j}^{2}+\lambda_{n}(\theta)$ of $\tilde{H}_{a}^{0}(\theta)$ are isolated and of finite multiplicity; with the above choice of $K$ they even become simple on $K$ for any fixed $n$ and $j=1$ if $a$ is small enough. They depend on $a$, of course, but one can perform the perturbation expansion with respect to $W(\theta):=$ $H_{a}(\theta)-H_{a}^{0}(\theta)$ around such running values. Mimicking the argument of [DE] we come to the following conclusion.

Lemma 2.3 Let $n_{0} \in \mathbb{N}$ and $E_{n_{0}}:=\left(\left(2 n_{0}-1\right) \pi / L\right)^{2}$. There exists a positive $a_{K, n_{0}}$ such that for all $a \in\left(0, a_{K, n_{0}}\right)$ and any $\theta \in K$, the spectrum of $\tilde{H}_{a}(\theta)$ below $E_{n_{0}}$ consists of exactly $n_{0}$ simple eigenvalues $\left\{\epsilon_{1, n}(a, \theta)\right\}_{1 \leq n \leq n_{0}}$. Moreover, the expansion

$$
\epsilon_{1, n}(a, \theta)=a^{-2} \kappa_{1}^{2}+\lambda_{n}(\theta)+\mathcal{O}(a)
$$

holds for each $n=1, \ldots, n_{0}$ uniformly in $K$.

Armed with these prerequisites, we can now formulate and prove the main result of this section.

Theorem 2.4 To any $E>0$ there is $a_{E}>0$ such that the spectrum of $-\Delta_{D}^{\Omega}$ is absolutely continuous in the interval $\left[0, a^{-2} \kappa_{1}^{2}+E\right]$ for all $a<a_{E}$.

Proof: By Lemma 2.1] we have to check that the eigenvalues of $\tilde{H}_{a}(\theta)$ are nowhere constant as functions of the quasimomentum $\theta$. Since they are real-analytic by Lemma 2.2, we have only to verify that each eigenvalue branch acquires at least two different values in the set $K$. Without loss of generality we can put $E=E_{n_{0}}$ for some $n_{0} \in \mathbb{N}$. Then there is just $n_{0}$ eigenvalues in $\left[0, a^{-2} \kappa_{1}^{2}+E\right]$, and consequently, there exists a $c_{E}>0$ such that $\left|\epsilon_{1, n}(a, \theta)-a^{-2} \kappa_{1}^{2}+\lambda_{n}(\theta)\right| \leq c_{E} a$ holds for each of them according to Lemma 2.3. Since the functions $\lambda_{n}(\cdot)$ are non-constant by [RS, Sec. XIII.16], the conclusion follows.

Remark 2.5 A similar perturbative argument shows that the spectrum is absolutely continuous at its bottom if $a$ is fixed and $\|\gamma\|_{\infty}$ is sufficiently small. 


\section{$3 \quad$ Leaky quantum wires}

Now we are going to consider the analogous problem for another class of operators. Let $\Gamma$ be the same $C^{4}$-smooth periodic curve in $\mathbb{R}^{d}$ and consider the generalized Schrödinger operator given by the formal expression

$$
H_{\alpha, \Gamma}=-\Delta-\alpha \delta(x-\Gamma) .
$$

Its meaning is different for different dimensions. If $d=2$ we regard it as the unique self-adjoint operator associated with the quadratic form

$$
q_{\alpha, \Gamma}[\psi]=\|\nabla \psi\|^{2}-\alpha \int_{\mathbb{R}}|\psi(\Gamma(s))|^{2} d s, \quad \psi \in W^{2,1}\left(\mathbb{R}^{2}\right),
$$

which is closed and below bounded by $[\mathrm{BEKS}]$; we suppose that the singular interaction is attractive, $\alpha>0$. The situation is more complicated in the three-dimensional case when $\operatorname{codim} \Gamma=2$. Following the construction given in [EK1] - see also [P0] for a more general background - one starts from the family of curves $\phi_{\Gamma}\left(\cdot, \rho, \vartheta_{0}\right)$, obtained by translating $\Gamma$ by $\rho\left(\cos \left(\vartheta_{0}\right), \sin \left(\vartheta_{0}\right)\right)$, which are used to determine the generalized boundary values of a function $f \psi \in W_{\text {loc }}^{2,2}\left(\mathbb{R}^{3} \backslash \Gamma\right) \cap L^{2}\left(\mathbb{R}^{3}\right)$ as the following limits

$$
\begin{aligned}
L_{0}(\psi)(s) & :=-\lim _{\rho \rightarrow 0} \frac{1}{\ln \rho} \psi\left(\phi_{\Gamma}\left(s, \rho, \vartheta_{0}\right)\right), \\
L_{1}(\psi)(s) & :=\lim _{\rho \rightarrow 0}\left[\psi\left(\phi_{\Gamma}\left(s, \rho, \vartheta_{0}\right)\right)+L_{0}(\psi)(s) \ln \rho\right] .
\end{aligned}
$$

We call $\Upsilon_{\Gamma}$ the family of those $\psi$ for which these limits exist a.e. in $\mathbb{R}$, are independent of $\vartheta_{0}$, and define a pair functions belonging to $L_{\text {loc }}^{2}(\mathbb{R})$. The sought operator is then defined as

$$
\begin{aligned}
H_{\alpha, \Gamma} \psi(x) & :=-\Delta \psi(x), \quad x \in \mathbb{R}^{3} \backslash \Gamma, \\
D\left(H_{\alpha, \Gamma}\right) & :=\left\{f \in \Upsilon_{\Gamma}: 2 \pi \alpha L_{0}(\psi)(s)=L_{1}(\psi)(s)\right\} .
\end{aligned}
$$

Being interested in strong coupling we suppose hereafter that the coupling parameter $\alpha$ is negative though $H_{\alpha, \Gamma}$ is well defined for all $\alpha \in \mathbb{R}$; recall that the two-dimensional $\delta$ interaction is always attractive [AGHH].

In an important particular case when $\Gamma$ is a straight line one uses separation of variables to show that the spectrum is absolutely continuous and 
covers the interval $[\zeta(\alpha), \infty)$, with the threshold given by the corresponding $(d-1)$-dimensional $\delta$ interaction eigenvalue,

$$
\zeta(\alpha):=\left\{\begin{array}{lll}
-\frac{1}{4} \alpha^{2} & \ldots & d=2 \\
-4 \mathrm{e}^{2(-2 \pi \alpha+\psi(1))} & \ldots & d=3
\end{array}\right.
$$

where $-\psi(1) \approx 0.577$ is the Euler number. This also illustrates that the strong coupling means $(-1)^{d} \alpha \rightarrow \infty$ for $d=2,3$. Due to the injectivity and periodicity assumptions the curve decomposes into a disjoint union of translates of the period cell $\Gamma_{\mathcal{P}}:=\Gamma \uparrow[0, L)$. Since $H_{\alpha, \Gamma}$ now acts in the whole Euclidean space, we need also a decomposition of the space $\mathbb{R}^{d}$ with the period cell

$$
\mathcal{P}:=\{\mathcal{L}+t b: t \in[0,1)\},
$$

where $\mathcal{L} \subset \mathbb{R}^{d}$ is a affine space which is not colinear with $b$. We denote by $b_{\perp}$ the component of $b$ in the direction orthogonal to $\mathcal{L}$; it follows that $b_{\perp} \neq 0$. It is important that the two decompositions are chosen in a consistent way, $\Gamma_{\mathcal{P}}=\mathcal{P} \cap \Gamma$. We will assume in addition that

(c) the restriction of $\Gamma_{\mathcal{P}}$ to the interior of $\mathcal{P}$ is connected.

It should be noted that the choice of a slab for $\mathcal{P}$ is made rather for convenience - see Remarks 3.5 below. We start again from the Floquet decomposition with respect to the Brillouin zone $\mathcal{B}:=\left[-\pi\left|b_{\perp}\right|^{-1}, \pi\left|b_{\perp}\right|^{-1}\right)$.

Lemma 3.1 There is a unitary $\mathcal{U}: L^{2}\left(\mathbb{R}^{d}\right) \rightarrow \int_{\mathcal{B}}^{\oplus} L^{2}(\mathcal{P}) d \theta$ such that

$$
\mathcal{U} H_{\alpha, \Gamma} \mathcal{U}^{-1}=\int_{\mathcal{B}}^{\oplus} H_{\alpha, \Gamma}(\theta) d \theta
$$

where the fibre operator satisfies periodic b.c. in the direction of $b$ acting as

$$
H_{a, \Gamma}(\theta)=(-i \nabla+\theta)^{2}-\alpha \delta(x-\Gamma),
$$

and the interaction term in $L^{2}(\mathcal{P})$ is interpreted in the above described sense, the quadratic form if $d=2$ and boundary conditions if $d=3$.

Proof: See [EY1] for $d=2$ and [EK2] for $d=3$.

It is easy to see that in distinction to the previous case the essential spectrum is non-empty and equals $\sigma_{\mathrm{ess}}\left(H_{a, \Gamma}(\theta)\right)=\left[\theta^{2}, \infty\right)$; we will be interested in the eigenvalues below its threshold. They are again real-analytic functions: 
Lemma 3.2 $\left\{H_{\alpha, \Gamma}(\theta): \theta \in \mathcal{B}\right\}$ is a type $A$ analytic family.

Proof: Similar to that of Lemma 2.2.

The role of the small tube width from the previous section is played here by strong coupling. While the wave function may be nonzero at large distances from $\Gamma$, it is localized in its vicinity as $(-1)^{d} \alpha \rightarrow \infty$. Then one can choose a tubular neighbourhood $\Omega$ of $\Gamma$ and estimate the operator in question from both sides by imposing the Dirichlet and Neumann condition at $\partial \Omega$. The exterior part does not contribute to the negative spectrum, while the part in $\Omega$ can be treated as in the previous section, with the additional $\delta$ interaction on the tube axis and different boundary conditions for the lower bound. The argument is thus more complicated, however, it was done in EY1 and EK2 with the following result.

Lemma 3.3 The number of isolated eigenvalues of $H_{\alpha, \Gamma}(\theta)$ exceeds any fixed $n \in \mathbb{N}$ as $(-1)^{d} \alpha \rightarrow \infty$. The $n$-th eigenvalue behaves asymptotically as

$$
\epsilon_{n}(\alpha, \theta):=\zeta(\alpha)+\lambda_{n}(\theta)+\left\{\begin{array}{l}
\mathcal{O}\left(\alpha^{-1} \ln \alpha\right) \\
\mathcal{O}\left(\mathrm{e}^{\pi \alpha}\right)
\end{array}\right\}
$$

in the strong coupling limit for $d=2,3$, respectively, uniformly in $\theta$, i.e. the error terms is for a fixed $n$ bounded in $\mathcal{B}$. Here $\lambda_{n}(\theta)$ means again the $n$-th eigenvalue of the operator $S(\theta)$ defined in the previous section.

The main result of this section then reads as follows.

Theorem 3.4 Under the stated assumptions, to any $E>0$ there exists an $\alpha_{E}>0$ such that the spectrum of the operator $H_{\alpha, \Gamma}$ is absolutely continuous in $(-\infty, \zeta(\alpha)+E]$ as long as $(-1)^{d} \alpha>\alpha_{E}$.

Proof: The argument is analogous to the one used for Theorem 2.4,

Remarks 3.5 (i) It is not always possible to choose $\mathcal{P}$ is the Cartesianproduct form, as we did above, which would satisfy the assumption (c). Counterexamples with a sufficiently entangled periodic $\Gamma$ are easily found. However, if we choose instead another period cell $\mathcal{P}$ with a smooth boundary, which is not a planar slab and for which the property (c) is valid, the argument modifies easily and the claim of Theorem 3.4 remains true. 
(ii) In the case $d=2$ such a "puzzle-like" decomposition can be always found. To see that, fold the plane into a cylinder of radius $|b| / 2 \pi$ so $\Gamma$ becomes a loop which encircles the cylindrical surface, dividing into two parts $\mathcal{C}^{ \pm}$which are disjoint apart of the common boundary; each of them is connected because $\Gamma$ has by assumption no self-intersections. Choosing a point at $\Gamma$, one can thus find two smooth semi-infinite curves in $\mathcal{C}^{ \pm}$, even straight from some point on, which go the two cylinder "infinities" without crossing $\Gamma$.

(iii) On the other hand, an analogous decomposition into translates of a suitable $\mathcal{P}$ satisfying the hypothesis (c) may not exist if $d=3$. It depends on the topology of $\Gamma$, a simple counterexample is given by a "crotchet-shaped" curve which enters $\mathcal{P}$ on its "left side" twice and leaves it once, and vice versa on the right, without being topologically equivalent to a line. We conjecture that the claim of Theorem 3.4 remains valid in such situations too, however, a different method is required to demonstrate it.

\section{Acknowledgments}

P.E. is grateful for the hospitality in Centre de Physique Théorique, C.N.R.S., where this work was started, and all the authors to Institut "Simion Stoilow" of the Romanian Academy, where it was finished. The research has been partially supported by GAAS under the contract A1048101.

\section{References}

[AGHH] S. Albeverio, F. Gesztesy, R. Høegh-Krohn, and H. Holden: Solvable Models in Quantum Mechanics, Springer, Heidelberg 1988.

[BSS̆] M.S. Birman, T.A. Suslina, and R.G. Shterenberg: Absolute continuity of the two-dimensional Schrödinger operator with delta potential concentrated on a periodic system of curves, Algebra i Analiz 12 (2000), 140-177; translated in St. Petersburg Math. J. 12 (2001), 535-567.

[BEKŠ] J.F. Brasche, P. Exner, Yu.A. Kuperin, and P. Šeba: Schrödinger operators with singular interactions, J. Math. Anal. Appl. 184 (1994), $112-139$.

[DE] P. Duclos and P. Exner: Curvature-induced bound states in quantum waveguides in two and three dimensions, Rev. Math. Phys. 7 (1995), 73-102. 
[EJK] P. Exner, A. Joye, and H. Kovař́ik: Magnetic transport in a straight parabolic channel, J. Phys. A34 (2001), 9733-9752.

[EK1] P. Exner and S. Kondej: Curvature-induced bound states for a $\delta$ interaction supported by a curve in $\mathbb{R}^{3}$, Ann. H. Poincaré 3 (2002), 967-981.

[EK2] P. Exner and S. Kondej: Strong-coupling asymptotic expansion for Schrödinger operators with a singular interaction supported by a curve in $\mathbb{R}^{3}$, math-ph/0303033

[EŠ] P.Exner, and P.Šeba: Bound states in curved quantum waveguides, J.Math. Phys. 30 (1989), 2574-2580.

[EY1] P. Exner, K. Yoshitomi: Band gap of the Schrödinger operator with a strong $\delta$-interaction on a periodic curve, Ann. H. Poincaré 2 (2001), 1139-1158.

[EY2] P. Exner and K. Yoshitomi: Persistent currents for 2D Schrödinger operator with a strong $\delta$-interaction on a loop, J. Phys. A35 (2002), 3479-3487.

[Ku] P. Kuchment: Floquet Theory For Partial Differential Equations, Birkhäuser Verlag, Basel 1993.

[Po] A. Posilicano: A Krein-like formula for singular perturbations of selfadjoint operators and applications, J. Funct. Anal. 183 (2001), 109-147.

[RS] M. Reed and B. Simon: Methods of Modern Mathematical Physics, IV. Analysis of Operators, Academic Press, New York 1978.

[SV] A.V. Sobolev and J. Walthoe: Absolute continuity in periodic waveguides, Proc. London Math. Soc. 85 (2002), 717-741.

[Yo] K.Yoshitomi: Band gap of the spectrum in peridically curved quantum waveguides, J. Diff. Eq. 142 (1998), 123-166. 